Letter to the editor

\section{Response to: Sleeve gastrectomy may double the risk of esophageal adenocarcinoma in morbidly obese patients}

We would like to thank Papadia et al. for their interest in our article, "Esophageal Adenocarcinoma After Sleeve Gastrectomy: Actual or Potential Threat? Italian Series and Literature Review" [1]. We also greatly appreciated their attempt at evaluating the relative risk (RR) of developing esophageal adenocarcinoma (EAC) in patients who undergo sleeve gastrectomy (SG). To do so, they extrapolated the reported number of SGs performed in Italy over the 3-year period (2012-2015) in which the EAC cases belonging to our series occurred. Papadia et al. [1] show how SG may raise the risk of developing EAC by an estimated 11-fold compared with the general population. Furthermore, they highlight how the RR of EAC in the SG subpopulation appears to be substantially greater compared with patients affected by morbid obesity (RR of 11.9 versus 4.8, respectively).

The authors also share our concern regarding the young age $(40.3 \pm 16.7 \mathrm{yr})$ and early presentation $(27.3 \pm 7.6$ mo) of EAC after surgery, pointing out how the progression from a normal esophageal mucosa to Barrett's esophagus (BE) generally befalls over a considerably longer timeframe in patients with GERD that did not have SG. This observation is supported by several studies published by our group demonstrating how the incidence of BE, 58 months after SG, is as high as $17.2 \%$ - consistent with other reported rates (i.e., 15\%-18.8\%) [2,3] -and is correlated to the increased presence of a biliary-type refluxate [4-6], likely to be responsible for the accelerated mucosal injury of the distal esophagus [7]. Emblematic is the case reported in literature of the patient who had a preoperatively diagnosed short-segment $\mathrm{BE}$ and who inevitably developed an EAC 36 months later [8]. Although a sporadically reported case, this further emphasizes not only the importance of performing a preoperative esophagogastroduodenoscopy (EGD) to detect any mucosal lesion, but also how BE should constitute an absolute contraindication to SG due to its innate risk of malignant evolution.
Finally, loss to follow-up still represents a major, longstanding issue after bariatric surgery, which contributes to precluding the chance of performing protocols of secondary prevention for the identification of any esophageal malignancy at its earliest stages. To this regard, close endoscopic surveillance is of paramount importance for a prompt detection. We also would like to reiterate the necessity of having international online registries, which could allow physicians to determine the actual incidence of EAC after SG and to comprehend its pathogenesis, management, and outcomes possibly better.

Despite this potential downside, SG persists as a safe and effective procedure for the cure of obesity and its co-morbid conditions, concurrently carrying low rates of long-term complications and nutritional deficiencies. Additionally, due to its greater technical simplicity and shorter operative time compared with other common bariatric procedures, SG is also the treatment of choice in the super-obese group, easily granting the option for revisional surgery (i.e., RYGB, one anastomosis gastric bypass [OAGB], duodenal switch [DS], single anastomosis duodeno-ileal sleeve [SADI-S]) in case of weight regain or insufficient weight loss. Hence, SG should yet be regarded as a fundamental and valuable bariatric operation to be performed after a thorough preoperative workup and a close endoscopic follow up.

Alfredo Genco, M.D. Lidia Castagneto-Gissey, M.D. Giovanni Casella, M.D., Ph.D. Department of Surgical Sciences Sapienza University of Rome Rome, Italy

\section{References}

[1] Genco A, Castagneto-Gissey L, Lorenzo M, Ernesti I, Soricelli E, Casella G. Esophageal adenocarcinoma after sleeve gastrectomy: actual or potential threat? Italian series and literature review. Surg Obes Relat Dis. Epub 2020 Nov 28. https://doi.org/10.1016/j.soard.2020.11.023 [Online ahead of print].

[2] Felsenreich DM, Kefurt R, Schermann M, et al. Reflux, sleeve dilation, and Barrett's esophagus after laparoscopic sleeve gastrectomy: longterm follow-up. Obes Surg 2017;27(12):3092-101. 
[3] Sebastianelli L, Benois M, Vanbiervliet G, et al. Systematic endoscopy 5 years after sleeve gastrectomy results in a high rate of Barrett's esophagus: results of a multicenter study. Obes Surg 2019;29(5): 1462-9.

[4] Genco A, Soricelli E, Casella G, et al. Gastroesophageal reflux disease and Barrett's esophagus after laparoscopic sleeve gastrectomy: a possible, underestimated long-term complication. Surg Obes Relat Dis 2017;13(4):568-74.

[5] Castagneto-Gissey L, Genco A, Del Corpo G, Badiali D, Pronio AM, Casella G. Sleeve gastrectomy and gastroesophageal reflux: a comprehensive endoscopic and $\mathrm{pH}$-manometric prospective study. Surg Obes Relat Dis 2020;16(11):1629-37.
[6] Soricelli E, Casella G, Baglio G, Maselli R, Ernesti I, Genco A. Lack of correlation between gastroesophageal reflux disease symptoms and esophageal lesions after sleeve gastrectomy. Surg Obes Relat Dis 2018;14(6):751-6.

[7] Kauer WK, Stein HJ. Role of acid and bile in the genesis of Barrett's esophagus. Chest Surg Clin N Am 2002;12(1):39-45.

[8] El Khoury L, Benvenga R, Romero R, Cohen R, Roussel J, Catheline J-M. Esophageal adenocarcinoma in Barrett's esophagus after sleeve gastrectomy: case report and literature review. Int J Surg Case Rep 2018;52:132-6.

https://doi.org/10.1016/j.soard.2021.02.010 